\title{
No-Show Rates and Associated Patient Factors in an Ophthalmology Resident Practice
}

\author{
Tavish Nanda, MD ${ }^{1}$ Aijin Wang, MS $\quad$ Luis M. Ducasse, MD ${ }^{1} \quad$ Royce W.S. Chen, MD ${ }^{1}$ \\ Jason Horowitz, $\mathrm{MD}^{1}$ \\ ${ }^{1}$ Edward S. Harkness Eye Institute, Columbia University Irving Medical \\ Center, New York, New York \\ 2 Department of Biostatistics, Columbia University Mailman School of \\ Public Health, New York, New York \\ Address for correspondence Tavish Nanda, MD, Edward S. Harkness \\ Eye Institute, 635 West 165th Street First Floor New York, NY 10032 \\ (e-mail: tn2290@cumc.columbia.edu).
}

J Acad Ophthalmol 2020;12:e67-e73.

\section{Abstract \\ Keywords \\ - quality improvement \\ - no-show rates \\ - follow-up \\ - appointment \\ - scheduling \\ - social services \\ - revenue}

Background In resident family medicine practices, a $>20 \%$ no-show rate is common, often resulting in suboptimal care. Few studies have evaluated resident ophthalmology practices in a similar fashion. The objective of this study was to determine no-show rates and factors related to missed appointments in a resident-based ophthalmology clinic.

Methods We reviewed 19,237 de-identified appointments over one academic year. Appointments were divided into general clinic visits and subspecialty visits (these two clinics function separately at our institution). The number of days between an appointment being made for a patient and the scheduled visit was defined as "lead time." Each appointment was labeled "show" or "no-show." Pertinent factors available in the analysis included lead time to appointment, patients age, month, time of day, practitioner training level (postgraduate years 2 to 4 , fellow), and proximity to holiday. Subspecialty clinics included oculoplastics clinic, strabismus clinic, neuro-ophthalmology clinic, uveitis clinic, cornea clinic, glaucoma clinic, and retina clinic. Generalized linear mixed effects models were fit on the no-show status with these factors.

Results The overall no-show rate for general clinic was 31.4\%. Longer lead time to appointment, younger age, afternoon appointments, new patients, December appointments, and lower residency level were associated with higher odds of noshow $(p \leq 0.05)$. The overall no-show rate for subspecialty clinic was $21.8 \%$. Longer lead time to appointment, younger age, and lower residency level were associated with higher odds of no-show $(p \leq 0.05)$. Patients were significantly less likely to show up for retina clinic, oculoplastics clinic, and glaucoma clinic.

Conclusion The overall no-show rate in our clinic was on the higher end of reported no-show values from other specialties. Patient status and the lead time to appointment play a significant role in patient compliance. The significance of other factors (patient age, subspecialty, time of day, appointment month, and resident level) have been found in previous studies but are not consistent factors across all clinical environments. received

October 22, 2019

accepted

April 13, 2020
DOI https://doi.org/

10.1055/s-0040-1712171. ISSN 2475-4757.
Copyright $\odot 2020$ by Thieme Medical Publishers, Inc., 333 Seventh Avenue, New York, NY 10001, USA. Tel: +1(212) 760-0888.
License terms

()ㅇㅇㅛ $\Theta$ 
Published no-show rates range from 3 to 80\%-across a large variety of specialties-with subspecialty outpatient clinics demonstrating higher no-show rates, often resulting in worsened health outcomes. ${ }^{1-11}$ One study at an ophthalmologyspecific resident practice demonstrated a $21.7 \%$ no-show rate. ${ }^{12}$ In comparison, faculty practices are demonstrably better, a factor that is often attributed to the socioeconomic status of patients who are seen by resident clinics. While ethnicity, gender, age, seasons, and time of day appear to be inconsistent factors in no-show rates, patients on Medicare, and in particular, Medicaid tend to be less likely to show. ${ }^{10,11,13,14}$

Naive overbooking is a common method in attempting to offset this no-show rate by applying multiple patients to the same time slots under the broad assumption that a certain percentage will not show. This method, however, results in an inconsistent work load and varied (often longer) patient waiting times, in turn producing increased clinic overtime, reduced patient satisfaction, and possibly even reduced quality of care. The consequence can be longer lead time to appointment scheduling, which in turn contributes to worsening no-show rates. ${ }^{3,11}$

Underutilization of clinic resources caused by excessive noshows will also result in reduced clinic revenue. One study estimated that $14 \%$ of daily revenue is lost by no-shows in a standard family practice, or-as a dollar amount-approximately \$196 dollars (2008 data) per no-show. ${ }^{9,14}$ Although no-shows can sometimes be offset by walk-in patients, the latter can be an unreliable source of substitute revenue. ${ }^{14}$

Anecdotally, our particular urban ophthalmology resident clinic suffers from a high no-show rate, even in the context of an automated telephone reminder program. The objective of this study was to review administrative records to more accurately quantify our no-show rate and identify factors that may result in poor compliance.

\section{Methods}

As part of a quality improvement (QI) initiative, we queried de-identified administrative records from a busy urban city resident clinic serving New York City, NY. All appointments were made with one of the following categories of physician: an ophthalmology resident physician ranging from 1 to 3 years of ophthalmology training, excluding internship (12 residents in total), two glaucoma fellows, three retina fellows, or one full-time staff optometrist. A total of 32,108 appointments were reviewed for 1 year from July 2017 to July 2018. Cancelled appointments, pediatrics patients, surgery follow-up patients, and inpatient consults were excluded, resulting in 19,237 appointments. This was further divided into general clinic visits and subspecialty visits $(13,123$ in general clinic and 6,110 in subspecialty clinic). These two clinics function independently at our institution and were analyzed separately. The general clinic is composed of ophthalmology residents spanning all years and an optometrist. The subspecialty clinic includes fellows and residents (of varying years). All subspecialty visits were referrals from the general clinic population. Residents in the subspecialty clinic share patients from a communal list, compared with the general clinic, in which residents have personal templates. There is no procedural difference in the scheduling process between first year ophthalmology residents, second year ophthalmology residents, third year ophthalmology residents, and fellows aside from differences in patient volume (second year residents, for example, spend several months on the inpatient consultation service).

The clinic scheduling program only fills appointments up to 135 days in advance. The number of days between an appointment being made for a patient and the scheduled visit was defined as "lead time to appointment." Each appointment was labeled as "show" or "no-show." New patients were patients with a first-time appointment with the eye clinic or patients who had not been seen for $>3$ years. New patients were only seen in general clinic; as subspecialty clinic requires a referral. All other patients were defined as "established." Clinical data such as disease severity or diagnosis were not collected. Other factors available for analysis included practitioner level, patient age, time of day, appointment month, and proximity to holidays. The prevalence of walk-in patients and the number of patients with multiple appointments was reviewed as well. Descriptive statistics were reported and generalized linear mixed effects models with both fixed and random effects, as well as polynomial terms, were used to determine the significance of the factors listed above in patient compliance.

\section{Results}

There were a total of 9,401 patients included in our study. A total of $38.3 \%$ were new patients. The overall no-show rate was $28.4 \%$ (31.4\% in general clinic and $21.8 \%$ in subspecialty clinic). The average number of patients scheduled for a full clinic day was 80.58 (standard deviation [SD]: 24.14), excluding Thursdays, which are usually lighter half-days due to structured academic time. In the general clinic, there were 4,785 total appointments for PGY4 residents, 1,408 for PGY3 residents, 3,445 for PGY2 residents, and 3,485 for optometry. In the subspecialty clinic, residents accounted for 4,590 visits, while fellows accounted for 1,520 visits. Each patient, on average, accounted for 2.05 (SD: 2.17, range: 1-30) scheduled visits in a year. Among 3,620 patients who had more than one appointment for the year, the median number of visits was 3 (Q1-Q3: 2-4). Two hundred and sixteen did not show up to any of their appointments.

Within the general clinic, new patients accounted for $43.7 \%$ of no-show appointments (show: median 71.91 days of lead time [Q1-Q3: 43.10-97.84], no-show: median 79.11 days of lead time [Q1-Q3: 60.85-102.13]). Among established patients, $26.8 \%$ of appointments were a no-show (show: median 56.95 days of lead time [Q1-Q3: 21.88-85.03], no-show: median 63.75 days of lead time [Q1-Q3: 38.00-93.14]). The respective no-show rates for the subspecialty clinics and the number of annual appointments are listed in - Table 1. Among all the subspecialties, strabismus appointments have the highest no-show rate and uveitis appointments have the lowest noshow rate. There were 1,410 walk-ins in the time period reviewed. This offset the number of no-shows by $25.8 \%$.

Generalized linear mixed effects models were fit for the general and subspecialty clinic data on the no-show status. 
Table 1 Prevalence of no-show by subspecialty clinic

\begin{tabular}{|l|l|l|}
\hline Specialty & $\begin{array}{l}\text { Of scheduled } \\
\text { visits }\end{array}$ & $\begin{array}{l}\text { No-show } \\
(\%)\end{array}$ \\
\hline Cornea & 511 & 23.9 \\
\hline Glaucoma & 1,591 & 20.6 \\
\hline Strabismus & 73 & 31.5 \\
\hline Neuro-ophthalmology & 409 & 27.1 \\
\hline Oculoplastics & 364 & 27.5 \\
\hline Retina & 2,498 & 20.6 \\
\hline Uveitis & 664 & 19.7 \\
\hline
\end{tabular}

Fixed effects included the potential factors listed in our methodology and anonymized patient ID was used as random effects to account for within subject correlation. From descriptive statistics, we discovered that among all the noncategorical variables, patient age variable had a nonlinear effect. Upon further model fitting, we decided to include a third-order polynomial term for patient age in the final model to account for the nonlinearity effect. Model results for both the general clinics and the subspecialty clinics are listed in -Table 2.

For general clinics, adjusting for all the other covariates, lead time to appointment, patient age, appointment month, time of day, patient status (new vs. established) and years of training for physicians are significantly associated with appointment no-show status. On average, for every 1 day increase in lead time to appointment, we expect to see an $0.8 \%$ increase in the odds of an appointment no-show ( $p<0.001)$; for an afternoon appointment, the odds of an appointment no-show are 1.127 times as large as the odds for a morning appointment $(p=0.003)$; for an established patient, the odds of an appointment no-show are 0.655 times as large as the odds of that for a new patient $(p<0.001)$; and lastly, for every 1 year increase in years of training for treating physicians, we expect to see $9.6 \%$ decrease in the odds of an appointment no-show $(p<0.001)$. Patient age is a significant predictor, though it has nonlinear effects. Appointment month is also significant in the model ( $p=0.022$; - Table 2B). Patients scheduled to see residents versus optometry did not demonstrate a significant difference in appointment adherence $(p=0.94)$.

For subspecialty clinics, lead time to appointment, patient age, and the level of training for physicians (residents vs. fellows) are significantly associated with appointment noshow status. Adjusting for all the other covariates, on average, for every 1-day increase in lead time to appointment, we expect to see a $1 \%$ increase in the odds of an appointment no-show $(p<0.001)$; and for appointments scheduled to a fellow, the odds of no-show are 0.773 times as large as the odds for a resident-scheduled appointment $(p=0.016)$. Patient age is a significant predictor, though it has nonlinear effects.

Table 2 Generalized linear mixed effects model output

\begin{tabular}{|c|c|c|c|c|}
\hline \multirow[b]{2}{*}{ Variable name } & \multirow[b]{2}{*}{ Adjusted OR } & \multicolumn{2}{|c|}{$95 \%$ confidence interval } & \multirow[b]{2}{*}{$p$-Value } \\
\hline & & Lower limit & Upper limit & \\
\hline \multicolumn{5}{|c|}{ 2A: For general clinic population (outcome $=$ no-show) } \\
\hline Lead time to appointment & 1.008 & 1.007 & 1.01 & $<0.0001$ \\
\hline $\mathrm{Age}^{\mathrm{a}}$ & & & & $<0.0001$ \\
\hline Month $^{\text {b: }}$ & & & & 0.022 \\
\hline Month: January & 0.946 & 0.756 & 1.183 & 0.6259 \\
\hline Month: February & 0.78 & 0.634 & 0.961 & 0.0193 \\
\hline Month: March & 0.847 & 0.698 & 1.027 & 0.0916 \\
\hline Month: April & 0.74 & 0.603 & 0.907 & 0.0038 \\
\hline Month: May & 0.681 & 0.555 & 0.834 & 0.0002 \\
\hline Month: June & 0.734 & 0.602 & 0.895 & 0.0022 \\
\hline Month: July & 0.815 & 0.681 & 0.975 & 0.0254 \\
\hline Month: August & 0.76 & 0.625 & 0.924 & 0.0059 \\
\hline Month: September & 0.792 & 0.648 & 0.966 & 0.0216 \\
\hline Month: October & 0.78 & 0.643 & 0.946 & 0.0117 \\
\hline Month: November & 0.823 & 0.674 & 1.005 & 0.0565 \\
\hline Time of day: afternoon & 1.127 & 1.04 & 1.221 & 0.0034 \\
\hline Patient Status: established & 0.655 & 0.596 & 0.719 & $<0.0001$ \\
\hline Proximity to holiday: no & 1.037 & 0.923 & 1.165 & 0.5412 \\
\hline Year of training & 0.904 & 0.859 & 0.952 & 0.0001 \\
\hline Resident vs. optometry ${ }^{c}$ & 0.995 & 0.884 & 1.121 & 0.9353 \\
\hline
\end{tabular}


Table 2 (Continued)

\begin{tabular}{|c|c|c|c|c|}
\hline \multirow[b]{2}{*}{ Variable name } & \multirow[b]{2}{*}{ Adjusted OR } & \multicolumn{2}{|c|}{ 95\% confidence interval } & \multirow[b]{2}{*}{$p$-Value } \\
\hline & & Lower limit & Upper limit & \\
\hline \multicolumn{5}{|c|}{ 2B: For subspecialty clinic population (outcome $=$ no-show) } \\
\hline Lead time to appointment & 1.01 & 1.008 & 1.013 & $<0.0001$ \\
\hline $\mathrm{Age}^{\mathrm{a}}$ & & & & 0.0003 \\
\hline Month & & & & 0.6883 \\
\hline Month: January & 0.888 & 0.63 & 1.251 & 0.4956 \\
\hline Month: February & 0.847 & 0.606 & 1.184 & 0.3315 \\
\hline Month: March & 1.036 & 0.749 & 1.432 & 0.8322 \\
\hline Month: April & 0.818 & 0.589 & 1.135 & 0.2292 \\
\hline Month: May & 0.782 & 0.553 & 1.106 & 0.2292 \\
\hline Month: June & 0.834 & 0.607 & 1.147 & 0.2644 \\
\hline Month: July & 0.806 & 0.604 & 1.074 & 0.1410 \\
\hline Month: August & 0.987 & 0.723 & 1.348 & 0.9354 \\
\hline Month: September & 0.827 & 0.589 & 1.161 & 0.2719 \\
\hline Month: October & 0.771 & 0.56 & 1.06 & 0.1096 \\
\hline Month: November & 0.881 & 0.631 & 1.23 & 0.4575 \\
\hline Time of day: afternoon & 1.105 & 0.913 & 1.338 & 0.3066 \\
\hline Subspecialty $^{\mathrm{d}}$ & & & & 0.2338 \\
\hline Subspecialty: EIC & 1.228 & 0.828 & 1.821 & 0.3079 \\
\hline Subspecialty: EIG & 1.462 & 1.028 & 2.078 & 0.0344 \\
\hline Subspecialty: EIM & 1.538 & 0.814 & 2.903 & 0.1846 \\
\hline Subspecialty: EIN & 1.26 & 0.841 & 1.889 & 0.2627 \\
\hline Subspecialty: EIO & 1.501 & 1.016 & 2.216 & 0.0413 \\
\hline Subspecialty: EIR & 1.471 & 1.078 & 2.008 & 0.0150 \\
\hline Proximity to holiday: no & 0.868 & 0.715 & 1.053 & 0.1506 \\
\hline Level of training: fellow & 0.773 & 0.627 & 0.954 & 0.0164 \\
\hline
\end{tabular}

Abbreviations: OR, odds ratio; EIC, cornea subspecialty; EIG, glaucoma subspecialty; EIN, neuro-ophthalmology subspecialty; EIO, oculoplastics subspecialty; EIR, retina subspecialty.

${ }^{\mathrm{a}} \mathrm{Age}$ variable was fit with a third-order polynomial term; therefore, we do not report odds ratio.

bWhen compared with December as the reference month.

${ }^{\mathrm{C}}$ For the sake of this analysis, we categorized optometrist as equivalent to a PGY2 resident.

${ }^{\mathrm{d}}$ When compared with uveitis clinic as the reference group.

When assessing the impact of subspecialty on no-show status in the generalized linear mixed effects model, using uveitis clinic as the reference group, we found no significance $(p=0.23)$. However, several subspecialty clinics specifically did appear to have significantly worsened patient compliance comparing to uveitis clinic. These include glaucoma $(p=0.03)$, oculoplastics $(p=0.04)$, and retina $(p=0.02)$.

\section{Discussion}

As expected, longer lead times are associated with higher odds of no-shows. ${ }^{12}$ Our overall no-show rate $(28.4 \%)$ is on the higher end of the spectrum of published no-show values when compared across other specialties and institutions. ${ }^{15}$ Previous studies on patients with chronic ophthalmologic disease have noted particularly poor adherence with appointments, and some suggest that the often subtle and slow-process of vision loss may result in a lack of urgency or motivation. ${ }^{15-19}$ Patients with Medicaid have also demonstrated consistently worse show rates in previous studies and may account for the higher no-show rate, as our patients rely predominantly on government insurance programs, though the exact percentage of government-based insurance versus other is not routinely recorded. ${ }^{11,20}$

Since patient status was a significant predictor in our model and established patient status is associated with a lower odds of no-show, continuity of care may be an important element in patient compliance. ${ }^{21}$ Getting the patient through the door in the first place and allowing familiarization with the clinic environment and their treating physician likely plays a role in appointment compliance. Older patients also had a lower odds of no-show. Though this appears counterintuitive on first 
glance, several previous studies have demonstrated a similar result. Smith and Yawn, for example, noted that patients older than 60 years are more likely to keep their appointments in a primary care setting. A large systematic review of 105 studies by Dantas et al found older age to be associated with appointment compliance across specialties. ${ }^{15}$ Presumably younger patients are healthier and less likely to suffer the health-related effects of missed follow-up. Additionally, the extent of planning required (home health aides, assisted travel) when mobility is an issue may tie older patients to their appointments.

Similar to some previous studies, there was a significant difference in no-shows for afternoon appointments and during the winter (especially December), though the significance of these two factors is not consistent in the literature. ${ }^{9,13,14,22}$ The higher odds of no-show for residents with fewer years of training has also been found in the published literature, and is attributed to a perceived or expected lack of clinical experience. ${ }^{11,23}$ We are limited in our ability to analyze patient adherence by socioeconomic status, ethnicity, gender, travel time/distance to clinic, or the specific weather for the day, as this information is not routinely collected. Previous studies have found several of these factors to be significant, with higher appointment compliance among Asian patients, private insurance holders, lower socioeconomic status, and increasing distance from care. ${ }^{9,13-16,22-31}$ Again, these factors are not consistent, with one study finding Asian patients to be less compliant, possibly due to language barrier issues or the lack of in-house translators. ${ }^{32}$ Another, counterintuitively, found closer distance to clinic ( $<3$ miles) to be associated with an increase in no-show. The authors attributed this to an increased reliance on public transportation, while those farther away may have access to personal vehicles. ${ }^{23}$

Of all the factors mentioned above, lead time to appointment and patient history of no shows remain the main determinant factors associated with poor compliance. Lead time, in particular, appears to have the greatest impact on no-show and is one of the few modifiable factors under the control of clinic administration. ${ }^{15,16}$

\section{Subspecialty}

It is unclear what factors resulted in increased no-shows in some subspecialty clinics versus others, though the chronic and often nonurgent nature of glaucoma and oculoplastics clinic complaints (i.e., ptosis, ectropion, and chalazion) may play a role. Uveitis demonstrated the highest prevalence of patient adherence possibly due to the significant discomfort and pain associated with acute uveitic events. In particular, Glaucoma is marred by historically poor medication compliance, a pattern that is unlikely to exclude appointment visits. ${ }^{32-35}$ Retina often requires more frequent follow-up and might result in patient fatigue. Another hypothesis is the fear and discomfort associated with intravitreal injections, which have become routine in these clinics.

In our analysis, retina and glaucoma had similar odds of no show (-Table 2B), while a prior ophthalmology-specific study found that patients with glaucoma demonstrated significantly higher odds of no show versus retina patients. In contrast, they attributed this to the use of invasive procedures, such as intravitreal injections, finding that those who received injections were actually more likely to show. The true outcome of these comparisons is yet to be determined, as their subanalysis was small and became nonsignificant when controlling for the type of retinal disease involved (diabetic retinopathy vs. macular degeneration). ${ }^{32}$ Our subspecialty analysis is similarly limited by its smaller sample size. When divided into individual clinics, strabismus clinic-for example-accounted for only 73 appointments in total ( - Table 1).

In the subspecialty setting, patients with poor health literacy regarding their disease process may be more likely to miss appointments. Patients who believe their eye disease is not a serious condition, or do not understand the projected trajectory of their disease, are less likely to comply. ${ }^{17-19}$ When answering questionnaires regarding their specific disease process, patients who answered $>50 \%$ incorrectly were more likely to no-show, even among college-educated patients. ${ }^{34}$ Interestingly, patients with worsened visual acuity, severe disease, or legal blindness were also less likely to show. This may be due to a reliance on added assistance (i.e., transportation and family members) or a consequence of habitual noncompliance, which has led to these end-stage disease states. ${ }^{34}$

Resident-specific practices are often focused around the differential diagnosis and treatment aspects of patient care, in part due to the teaching methodology required to train residents to be well-versed ophthalmologists. A re-emphasis on the importance of patient education as well may be paramount to improving appointment compliance and ultimately patient outcomes. A process that maximizes patient show rates could provide residents with the predictability and time required to counsel patients at the end of each visit.

\section{Reminder Programs}

In previous surveys on patients who missed their appointments both in primary care and ophthalmology, forgetfulness (17.6-35.5\%) and miscommunication/clerical error (27.3-31.5\%) were the two most common reasons, with work commitments and health insurance as rather minimal impedances. ${ }^{20,36-45}$ While our clinic employs an automated telephone reminder program 48 hours prior to a scheduled appointment, it is unclear how efficacious this process is.

The literature agrees that a reminder program is an important element in reducing no-shows, but it appears as though automated telephone programs may demonstrate less efficacy (as little as $0.5 \%$ improvement) when compared with SMS messaging or real-person telephone reminders, especially those that require a patient to interact (i.e., call back to cancel or acknowledge an appointment by text). ${ }^{9,13,20,36-45}$ The main barrier to these methods is the difficulty in contacting patients, in part due to inaccurate or incomplete registration information. ${ }^{12}$ An emphasis on gathering this accurately, even without changing reminder methodology, may significantly reduce no-show rates. Targeted reminders for those with multiple factors associated with no-shows (i.e., a young patient with an afternoon appointment in December) or those who require close follow-up may also reduce no-shows with minimal strain on front desk 
staff. Simple solutions, such as day-to-day variation in the time the phone call is made (to account for those who are unavailable at certain times) or repeat reminders for "highrisk" patients, may result in added improvement, with little additional investment. ${ }^{20,43}$

\section{Scheduling Solutions}

Algorithmic scheduling is a new and interesting concept in the era of pattern modeling that utilizes no show probabilities to automatically build more efficient scheduling templates. While this methodology is outside the scope of this study, in theory, the significant factors listed above would be weighted automatically, and each patient would be assigned a numerical value associated with their chance of showing up to their appointment. A more robust prediction model would include socioeconomic data, diagnosis, and historical appointment compliance. Such a system is ideally flexible, changing with every ensuing visit based on whether or not a patient showed and using that data continuously to tailor future templates.

One study demonstrated the efficacy of this method by assigning a value to each patients' chance of showing up to their appointment, based on demographic information and historical show rates, with a $63 \%$ improvement in physician utilization. In this study, the clinical template was overbooked $83 \%$ of the time, underbooked $19 \%$ of the time, and increased patient capacity by $12 \%^{13}$

Rather than naive overbooking, certain templates with "less compliant" patients would have more open slots, while others with more compliant patients would be capped. Our study supports the advantage of such a program. For example, 216 patients with more than one appointment during the year did not show to any of the appointments and could be placed on "busier" templates. Even without extensive clinical data, the factors analyzed here can help tailor scheduling templates to maximize accuracy and reduce the burden on both residents and patient wait times. This method of patient scheduling appears generalizable to any clinic that has basic billing or demographic patient data.

Another possible model is the use of a 2-week "open access" schedule, in which patients can consistently book appointments within a 2-week time-frame if needed. This improves the predictability of a patient showing up and reduces periods of under or overstaffing. The use of this concept in an ophthalmology residency practice, in simulation, has been estimated to reduce no-show rates by as much as $60 \% .{ }^{12}$ Both new and established patients would benefit from any scheduling system that reduces lead time to appointment.

\section{Social Services}

The vast majority of our catchment area is comprised predominantly of an immigrant community from the Dominican Republic. This may bias our no-show rates, as many of these patients have special circumstances, such as undocumented status, repeat visits abroad, lack of social services assistance, and limited contact information. ${ }^{20}$ Currently, our clinic and many other ophthalmology clinics do not employ social work staff, as these issues are not considered relevant to eye care. In comparison, a previous study on a primary care practice with a remarkable $6.7 \%$ (vs. usual $>25 \%$ ) no-show rate attributed their significantly above average appointment compliance to the full-time employment of two social workers. ${ }^{11}$ Consideration should be given to the employment of at least one social services provider, especially in the context of providing referral for low vision resources.

\section{Revenue}

One study on a primary care resident practice found that over 250 days, the estimated loss of revenue from no-shows (31.1\%) reached $\$ 353,008$ ( $\sim 57$ revenue per visit). This was in the setting of a high walk-in to no-show rate (60\%) to compensate for daily no-show numbers. ${ }^{14}$ Our institution appears less resilient, with walk-ins offsetting total no shows by only $25.8 \%$. With the practice of overbooking, however, it is unclear how much revenue is actually lost due to noshows. It may be that the practice of overbooking compensates appropriately and fulfills the clinic template to capacity (albeit without the benefit of predictability). The concept of wait time, patient satisfaction, inefficient staffing, and its influence on revenue is an important future study that will better determine the cost-benefit of investing in an algorithmic scheduling system. In the one study estimating the cost of development and implementation of an algorithmic scheduling process, the break-even point-when taking into account the revenue saved from reduced no-showswas approximately 2 months (using data from mid-western clinics), after which this approach became profitable. ${ }^{13}$

\section{Conclusion}

Most outpatient clinics try to target a no-show rate of 5 to $10 \% .^{12}$ One can gather from the literature cited that few patient characteristics can be reliably applied to all clinical environments. ${ }^{15}$ In the era of precision medicine, there appears to be a space for "targeted" quality improvement, in which solutions are built around patterns and trends for a specific clinic's experience (i.e., that appointment no show is not simply a random event). ${ }^{15,22}$ This study is limited by its retrospective nature and lack of in-depth disease severity analysis. Further study on distance traveled, familial burden, hospitalization rates, and coexisting medical problems, to name a few, may better characterize the findings listed above. ${ }^{13}$ Our clinic also does not build a scheduling template outside of 135 days, limiting our ability to analyze 6-month and annual follow-up show rates. This study does, however, demonstrate the complexity and importance of improving patient scheduling, and the possible utility of predictive factors in assessing patient compliance.

\section{Note}

This study was presented, in part, at The Association for Vision and Research Annual Meeting 2019.

\section{Funding}

None.

Conflict of Interest

None declared. 


\section{References}

1 Sharp DJ, Hamilton W. Non-attendance at general practices and outpatient clinics. BMJ 2001;323(7321):1081-1082

2 Ferguson S, Kokesh J. Remote Otolaryngology Services: A Cost Comparison Study. AK: The Alaska Federal Health Care Network; 2005

3 Rust CT, Gallups NH, Clark WS, Jones DS, Wilcox WD. Patient appointment failures in pediatric resident continuity clinics. Arch Pediatr Adolesc Med 1995;149(06):693-695

4 Johnson BJ, Mold JW, Pontious JM. Reduction and management of no-shows by family medicine residency practice exemplars. Ann Fam Med 2007;5(06):534-539

5 Bennett KJ, Baxley EG. The effect of a carve-out advanced access scheduling system on no-show rates. Fam Med 2009;41(01): 51-56

6 Dreiher J, Goldbart A, Hershkovich J, Vardy DA, Cohen AD. Factors associated with non-attendance at pediatric allergy clinics. Pediatr Allergy Immunol 2008;19(06):559-563

7 Cohen AD, Dreiher J, Vardy DA, Weitzman D. Nonattendance in a dermatology clinic-a large sample analysis. J Eur Acad Dermatol Venereol 2008;22(10):1178-1183

8 Lehmann TN, Aebi A, Lehmann D, Balandraux Olivet M, Stalder H. Missed appointments at a Swiss university outpatient clinic. Public Health 2007;121(10):790-799

9 Kheirkhah P, Feng Q Travis LM, Tavakoli-Tabasi S, Sharafkhaneh A. Prevalence, predictors and economic consequences of no-shows. BMC Health Serv Res 2016;16(01):13

10 Hixon AL, Chapman RW, Nuovo J. Failure to keep clinic appointments: implications for residency education and productivity. Fam Med 1999;31(09):627-630

11 Weingarten N, Meyer DL, Schneid JA. Failed appointments in residency practices: who misses them and what providers are most affected? J Am Board Fam Pract 1997;10(06):407-411

12 McMullen MJ, Netland PA. Lead time for appointment and the noshow rate in an ophthalmology clinic. Clin Ophthalmol 2015; 9:513-516

13 Daggy J, Lawley M, Willis D, et al. Using no-show modeling to improve clinic performance. Health Informatics J 2010;16(04): 246-259

14 Moore CG, Wilson-Witherspoon P, Probst JC. Time and money: effects of no-shows at a family practice residency clinic. Fam Med 2001;33(07):522-527

15 Dantas LF, Fleck JL, Cyrino Oliveira FL, Hamacher S. No-shows in appointment scheduling - a systematic literature review. Health Policy 2018;122(04):412-421

16 Norris JB, Kumar C, Chand S, Moskowitz H, Shade SA, Willis DR. An empirical investigation into factors affecting patient cancellations and no-shows at outpatient clinics. Decis Support Syst 2014;57:428-443

17 Kosoko O, Quigley HA, Vitale S, Enger C, Kerrigan L, Tielsch JM. Risk factors for noncompliance with glaucoma follow-up visits in a residents' eye clinic. Ophthalmology 1998;105(11):2105-2111

18 Lee BW, Sathyan P, John RK, Singh K, Robin AL. Predictors of and barriers associated with poor follow-up in patients with glaucoma in South India. Arch Ophthalmol 2008;126(10):1448-1454

19 Murakami Y, Lee BW, Duncan M, et al. Racial and ethnic disparities in adherence to glaucoma follow-up visits in a county hospital population. Arch Ophthalmol 2011;129(07):872-878

20 Kaplan-Lewis E, Percac-Lima S. No-show to primary care appointments: why patients do not come. J Prim Care Community Health 2013;4(04):251-255

21 Taplin SH, Barlow WE, Ludman E, et al. Testing reminder and motivational telephone calls to increase screening mammography: a randomized study. J Natl Cancer Inst 2000;92(03):233-242

22 King A, David D, Jones HS, O'Brien C. Factors affecting nonattendance in an ophthalmic outpatient department. J R Soc Med 1995;88(02):88-90
23 Smith CM, Yawn BP. Factors associated with appointment keeping in a family practice residency clinic. J Fam Pract 1994;38(01):25-29

24 Hagerman GA. Testing the mailed appointment reminder in family practice. J Fam Pract 1978;7(01):199-201

25 Garuda SR, Javalgi RG, Talluri VS. Tackling no-show behavior: a market-driven approach. Health Mark Q 1998;15(04):25-44

26 Miller AJ, Chae E, Peterson E, Ko AB. Predictors of repeated "noshowing" to clinic appointments. Am J Otolaryngol 2015;36(03): 411-414

27 Hamilton W, Round A, Sharp D. Patient, hospital, and general practitioner characteristics associated with non-attendance: a cohort study. Br J Gen Pract 2002;52(477):317-319

28 Bean AG, Talaga J. Predicting appointment breaking. J Health Care Mark 1995;15(01):29-34

29 Bickler CB. Defaulted appointments in general practice. J R Coll Gen Pract 1985;35(270):19-22

30 Oppenheim GL, Bergman JJ, English EC. Failed appointments: a review. J Fam Pract 1979;8(04):789-796

31 Bottomley WW, Cotterill JA. An audit of the factors involved in new patient non-attendance in a dermatology out-patient department. Clin Exp Dermatol 1994;19(05):399-400

32 Thompson AC, Thompson MO, Young DL, et al. Barriers to followup and strategies to improve adherence to appointments for care of chronic eye diseases. Invest Ophthalmol Vis Sci 2015;56(08): 4324-4331

33 Schwartz GF. Compliance and persistency in glaucoma follow-up treatment. Curr Opin Ophthalmol 2005;16(02):114-121

34 Kholdebarin R, Campbell RJ, Jin YP, Buys YM, Canadian Compliance Study Group. Multicenter study of compliance and drop administration in glaucoma. Can J Ophthalmol 2008;43(04):454-461

35 Potamitis T, Chell PB, Jones HS, Murray PI. Non-attendance at ophthalmology outpatient clinics. J R Soc Med 1994;87(10): 591-593

36 Cialdini RB. The science of persuasion. Sci Am 2001;284(02): 76-81

37 Brannan SO, Dewar C, Taggerty L, Clark S. The effect of short messaging service text on non-attendance in a general ophthalmology clinic. Scott Med J 2011;56(03):148-150

38 Taylor NF, Bottrell J, Lawler K, Benjamin D. Mobile telephone short message service reminders can reduce nonattendance in physical therapy outpatient clinics: a randomized controlled trial. Arch Phys Med Rehabil 2012;93(01):21-26

39 Dockery F, Rajkumar C, Chapman C, Bulpitt C, Nicholl C. The effect of reminder calls in reducing non-attendance rates at care of the elderly clinics. Postgrad Med J 2001;77(903):37-39

40 Parikh A, Gupta K, Wilson AC, Fields K, Cosgrove NM, Kostis JB. The effectiveness of outpatient appointment reminder systems in reducing no-show rates. Am J Med 2010;123(06):542-548

41 Fairhurst K, Sheikh A. Texting appointment reminders to repeated non-attenders in primary care: randomised controlled study. Qual Saf Health Care 2008;17(05):373-376

42 Hasvold PE, Wootton R. Use of telephone and SMS reminders to improve attendance at hospital appointments: a systematic review. J Telemed Telecare 2011;17(07):358-364

43 Roberts N, Meade K, Partridge M. The effect of telephone reminders on attendance in respiratory outpatient clinics. J Health Serv Res Policy 2007;12(02):69-72

44 Koshy E, Car J, Majeed A. Effectiveness of mobile-phone short message service (SMS) reminders for ophthalmology outpatient appointments: observational study. BMC Ophthalmol 2008;8(01):9

45 Roth JP, Kula TJ Jr, Glaros A, Kula K. Effect of a computer-generated telephone reminder system on appointment attendance. In Seminars in orthodontics 2004 Sep 1 (Vol. 10, No. 3, pp. 190-193). WB Saunders. Available at: https://www.televox.com/downloads/Case_Study_Seminars_in_Orthodontics.pdf. Accessed April 24, 2020 